\title{
A Methodology for Reducing Bench Strength in Information Technology Companies
}

\author{
Boby John \\ Technical Officer, Statistical Quality Control \& Operations Research Unit, Indian Statistical Institute, 8th Mile, Mysore \\ Road, Bangalore, Karnataka State, India - 560 059. E-mail: boby@isibang.ac.in
}

Project Management

Received July 8, 2014; received revision March 28, 2015; accepted April 25, 2015

Available online May 11, 2015

\begin{abstract}
One of the major risks in information technology (IT) project execution is the non-availability of resources with required skill sets to meet the project demands. To mitigate this risk, almost every IT organization maintains a portion of their resources on bench. The bench strength reduces the delay in starting the project due to the nonavailability of skilled resources. But bench strength or buffer is cost to the company. A medium scale Indian IT company incurs around USD 142,347 monthly on maintaining the bench strength. Hence this study is undertaken to develop a methodology to reduce the bench strength in IT companies. The proposed methodology is to obtain an optimum mix of resources from bench and through recruitment to meet the demands at a minimum cost. This is done by formulating the problem as an integer programming problem. The problem aims to minimize the total cost of obtaining the skilled resources without violating the constraints on demand, bench strength availability and recruitment lead time. The problem can be solved using Microsoft excel solver function or any other optimization packages like CPLEX, Gurobi, etc. A case study on the application of proposed methodology is also discussed in the paper. The case study showed that the proposed methodology is superior to the existing practice of maintaining large bench strengths to meet the demands for resources with various skills.
\end{abstract}

Keywords: Bench strength, polynomial regression, constraint optimization, integer programming.

\section{Introduction}

Information technology (IT) has enabled organizations to improve productivity, reduce cost and time in manufacturing products, delivering services, etc. There is great demand for IT applications to manage various business processes. The business of IT companies is to take up the projects of developing information technology applications. The success of IT projects are often judged based on their performance with respect to cost, quality and schedule (Jalote, 2000). Hence identification and mitigation of risks are very important for the success of IT projects (Chua et al., 1999).

The risks involved in project execution can be mitigated to a great extend by the application of appropriate knowledge, skills, processes and techniques (Porananond and Thawesaengskulthai, 2014). Since software development is a human activity and also due to the rapidly changing nature of information technology field (Bouras and Bendak, 2014), the most critical risk in IT projects is the non-availability of resources with the correct skills at the right time. One of the ways to manage the aforementioned resource constraint (Nguyen and Chua, 2014) is to maintain large buffer or bench strength.

At any point of time, information technology (IT) companies have a portion of their resources on bench. The organizations retain the resources on bench to ensure that whenever a new project deal is materialized, there is no shortage of the resources with required skill set to commence the work. The bench strength reduces the delay in starting the project due to the non-availability of skilled resources (Narayanan and Swaminathan, 2007). But over capacity is a cost to any organization (Lennartsson and Björnfot, 2012). Generally a medium scale Indian IT company maintains bench strength of 5 to $6 \%$. The approximate cost of maintaining this bench strength is USD 142,347 per month. Hence this study is undertaken to develop a methodology to reduce the bench strength for information technology companies.

The remaining part of this paper is arranged as follows: the methodology is explained in session 2, a case study based on the proposed methodology is given in session 3 , the results are presented in session 4 and session 5 discusses the conclusion.

\section{Methodology}

The proposed methodology aims to ensure the availability of required number of resources with right skills whenever the resources are required at a minimum cost. The resource can be made available by keeping a pool of them in the bench or by recruiting them whenever the need arises. Both involve costs, namely the recruitment cost and cost of maintaining the bench strength. The recruitment channel may have a time lag also. Hence the suggested methodology is to select an optimum mix of resources from bench as well as through recruitment so 
that the demand will be met on time at a minimum total cost. The total cost is the sum of recruitment cost and bench strength cost.

A lot of problems related to optimum assignment of employees to jobs have been solved in the past. The major works published in the recent past are the solution to the problem of assigning employees to about 86 gas stations distributed all over Kuwait using mixed integer programming (Al-Yakoob and Sherali, 2007), development of a mixed integer linear programming model for a flow shop with multi-processor workstations (Huq et al., 2004), the problem of scheduling part-time work force using integer programming with the objective of maximizing employee satisfaction subject to meeting the demand requirements of each shift (Mohan, 2008), solution to employee production scheduling with uncertain up times of equipment and production rates using Bayesian forecasting and stochastic programming techniques (Morton and Popova, 2004) and the solution to the constrained scheduling of employee resourcing for a mall type shop using genetic algorithm (Brezulianu et al., 2009).

In this paper, the author discusses a slightly different problem, the problem of optimization of the buffer or bench strength. In other words, the problem of finding the right balance of the cost of keeping employees on bench and the cost for hiring new ones for IT organizations. The formulation of the problem is given below

Let

$x_{i}$ be the number of resources needed on bench with skill set $i, i=1,2, \ldots k, k$ is the number of different skill set required

$y_{i}$ be the number of resources to be recruited with skill set $i, i=1,2, \ldots, k$

$d_{i}$ be the number of resources required (demand) with skill set $i, i=1,2, \ldots, k$

$c_{i}$ be the cost for an employee on bench with skill set $i, i$ $=1,2, \ldots, k$

$r_{i}$ be the recruitment cost for an employee with skill set $i$, $i=1,2, \ldots, k$

$t_{i}$ be the lead time to recruit a resource with skill set $i, i=$ $1,2, \ldots, k$

$T_{i}$ be the maximum time available for recruitment of resources with skill set $i, i=1,2, \ldots, k$

$A_{i}$ be the number of resources available on bench with skill set $i, i=1,2, \ldots, k$

The problem is to find out the optimum values of $x_{i} \& y_{i}, i$ $=i=1,2, \ldots, k$ which would minimize the total cost and satisfy the constraints on demand, time \& availability.

The problem is to

Minimize $\mathrm{z}=\sum_{i=1}^{k} c_{i} x_{i}+\sum_{i=1}^{k} r_{i} y_{i}$

Subject to

1. Demand constraints

$$
\begin{aligned}
& x_{i}+y_{i} \geq d_{i}, \\
& i=1,2, \ldots, k
\end{aligned}
$$

2. Lead Time to Recruitment constraints

$$
t_{i} y_{i} \leq T_{i}, i=1,2, \ldots, k
$$

3. Bench Strength Availability Constraints

$$
x_{i} \leq A_{i}, i=1,2, \ldots, k
$$

4. Non Zero Constraints

$$
\begin{aligned}
& x_{i} \geq 0 \\
& y_{i} \geq 0
\end{aligned}, i=1,2, \ldots, k
$$

5. Integer Constraints

$$
\begin{aligned}
& x_{i}=\text { int eger } \\
& y_{i}=\text { int eger }
\end{aligned}, i=1,2, \ldots, k
$$

The aforementioned optimization problem (1) subject to the constraints (2) to (6) is an integer programming problem (Taha, 1987; Hillier \& Lieberman, 2008; Atamturk and Savelsbergh, 2005). The integer programming is widely used in industries to solve optimization problems like resource allocation in production planning ( $\mathrm{Li}$ and Sun, 2006), reliability redundancy optimization (Coelho, 2009), process scheduling (Floudas and Lin, 2005), etc. The integer programming problem (1) can be solved using the Microsoft excel's solver function. The MS Excel solver uses generalized reduced gradient algorithm to solve constrained optimization problems (Fylstra et al., 1999). Lot of studies have been published on the applications of MS Excel solver in solving industrial problems (Souliman, et al., 2010; Dasgupta, 2008; Fang, 2006; Brown, 2006).

The solution of the problem (1) would provide the optimum number of resources with various skills to be recruited as well as selected from bench to meet the demand at a minimum cost. A case study on the application of the methodology is given in the next session.

\section{Case Study}

This study is carried out in an Indian medium scale information technology company. Currently the total strength of the company is 3950, out of which 216 resources are on bench. The skill wise distribution of bench strength is given in Fig. 1.

The problem is to find out the optimum number of resources with various skills to be maintained in the bench to meet the upcoming month's demand. To formulate the problem, the monthly data on the following attributes is collected and is given in Table 1.

1. Skill type

2. Bench strength

3. Estimated demand of resources for the upcoming month

4. Monthly cost of an employee on bench

5. Recruitment cost per employee

6. Estimated time for recruitment (in days) 
Table 1. Bench strength

\begin{tabular}{ccccccc}
\hline Skill & Skill Type & $\begin{array}{c}\text { Bench } \\
\text { Strength }\end{array}$ & $\begin{array}{c}\text { Estimated } \\
\text { Demand }\end{array}$ & Bench Cost(\$) & $\begin{array}{c}\text { Recruitment } \\
\text { Cost (\$) }\end{array}$ & $\begin{array}{c}\text { Recruitment } \\
\text { Lead Time }\end{array}$ \\
\hline Testing & Special & 65 & 30 & 640 & 30 & - \\
EAS SAP & Special & 43 & 28 & 800 & 33 & - \\
ADM & Special & 32 & 22 & 933 & 42 & - \\
.Net & General & 39 & 8 & 480 & 25 & 12 \\
Java & General & 37 & 12 & 480 & 21 & 18 \\
Total & & 216 & 100 & 3333 & 151 & \\
\hline & & & & & Testing
\end{tabular}

The discussions with the company professionals revealed that the resource with general skills will be available in the job market and can be recruited within a specified number of days. But the resources with special skills (namely testing, EAS SAP \& ADM) may not be readily available and the time to recruit the resources with special skills varies with the number of resources required. Hence it is decided to study the relationship between the number of resources with special skills and the lead time to recruitment. The scatter plots of number of resources versus the recruitment lead time for different special skills are plotted using R statistical package (Zhao, 2012). The scatter plots are given in Fig. 2, Fig. 3 and Fig. 4. The scatter plot is a visualization of correlation between two variables. The correlation measures the strength of relationship or association between the variables (Crawford, 2006; Zou et al., 2003).

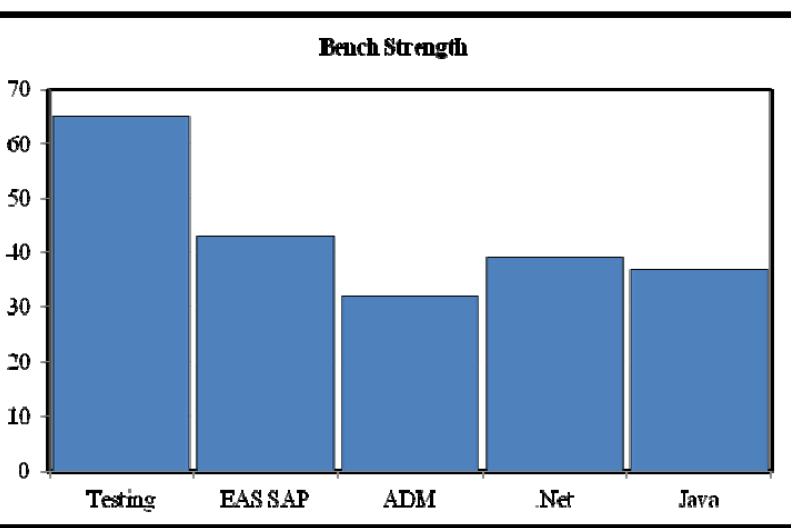

Fig. 1. Current bench strength

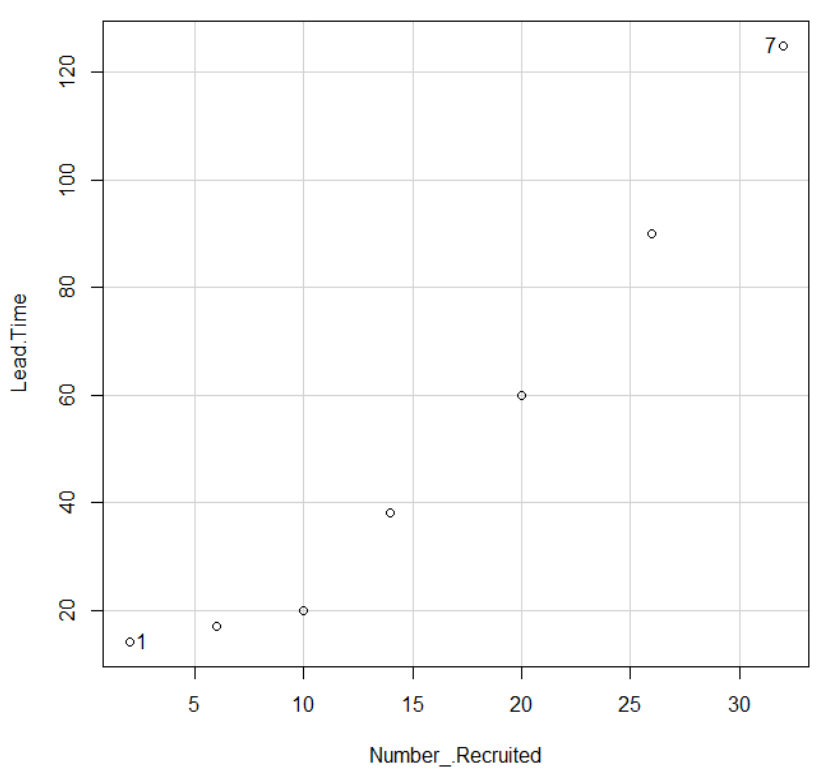

Fig. 2. Scatter plot for skill: Testing

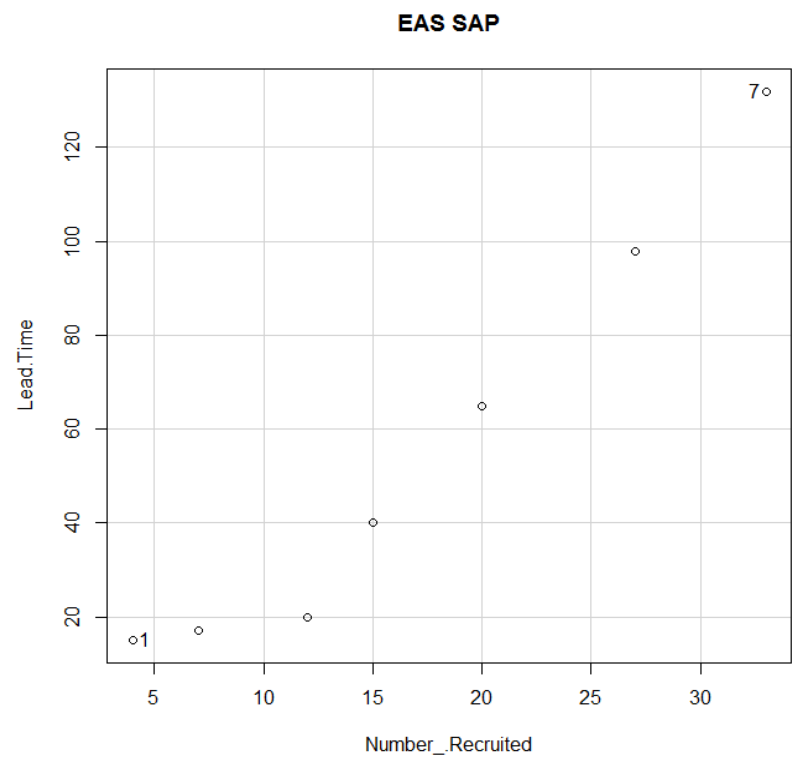

Fig. 3. Scatter plot for skill: EAS SAP 
The analysis of scatter plots (Czarnigowska and Sobotka, 2014; Boby and Kadadevaramath, 2013) revealed that the relationship between the lead time for recruitment and number of resources required are not linearly related. The Fig. 2, Fig. 3 and Fig. 4 show that when the number of resources required are less (may be up to 15 ), the lead time to recruitment gradually increases as the number of resources increases. But when the number of resources required is more than 15 , the lead time to recruit increases rapidly with the increase in number of resources. When the relationship between the variables is not linear, one of the ways to fit a model is to use polynomial regression technique (Montgomery et al., 2012). In polynomial regression, along with linear terms, square, cube, etc. terms are also fitted to account for the curvature in the relationship between variables (Crawley, 2012). Hence the models for estimating the recruitment lead time for resources with special skills, namely testing, EAS SAP and ADM are developed using polynomial regression. The major steps in model development are the following. The explanatory variable, the number of resources required is standardized using (7)

$$
z=\frac{(\text { value }- \text { mean })}{s \tan \text { dard deviation }}
$$

where mean is the arithmetic average and standard deviation is the standard deviation of the explanatory variable. Then regression models for lead time are developed in terms of linear, quadratic and cubic terms of standardized explanatory variable. The model coefficients and corresponding $\mathrm{p}$ values are given in Table 2. The Table 2 showed that the cubic term is insignificant for all the three models as the corresponding p value $>0.05$ (Draper \& Smith, 2003). Hence only linear and quadratic (square) terms are retained in the models. The $\mathrm{R}^{2}$, adjusted $\mathrm{R}^{2}$ and standard error $\mathrm{S}$ for models with linear and quadratic terms are given in Table 3 . The Table 3 shows that the quadratic model is better than the linear model as $\mathrm{R}^{2}$ and adjusted $\mathrm{R}^{2}$ are high and standard error $\mathrm{S}$ is small. This is true for the models for all the three skill sets.

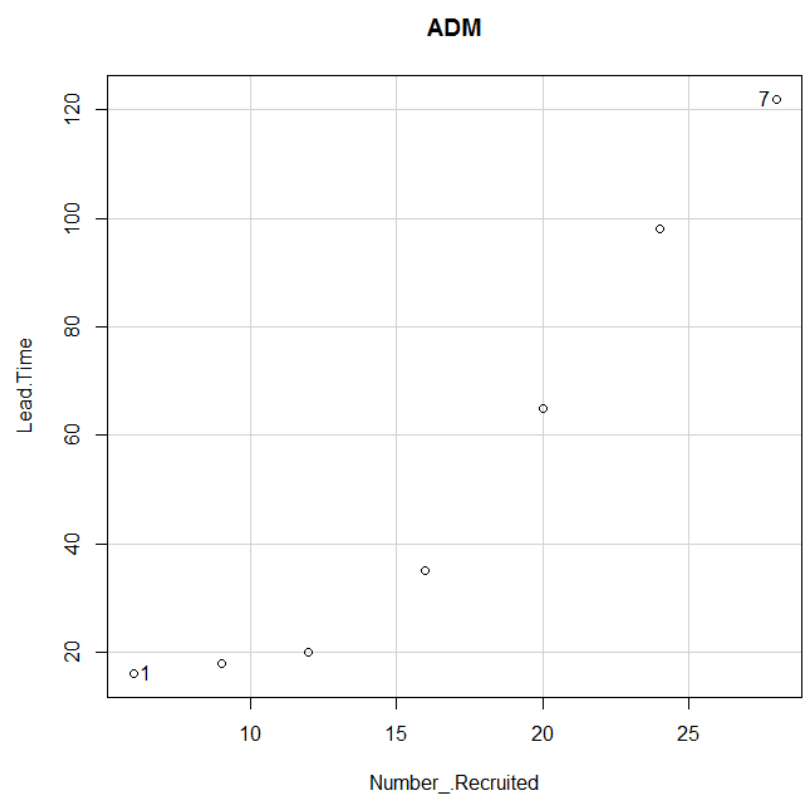

Fig. 4. Scatter plot for skill: ADM
The $F$ statistic and corresponding $p$ values of analysis of variance for the quadratic models is given in Table 4 . The Table 4 shows that the $p$ value $<0.05$ for all the three models and hence the regression models are reasonably good (Montgomery and Runger, 2007). Moreover the residuals are approximately normally distributed and there is no trend or pattern in residuals versus fitted values or residuals versus order of the data (Czarnigowska and Sobotka, 2014). Hence it is decided to use the quadratic models to estimate the recruitment lead time for resources with special skill sets. The model coefficients in terms of original explanatory variable, namely number of resources required, is given in Table 5.

Table 2. Coefficients and p values of models

\begin{tabular}{cccc}
\hline \multirow{2}{*}{ Skill } & Model & Coefficient & p value \\
\hline \multirow{3}{*}{ Testing } & Intercept & 41.5794219 & 0.000109 \\
& Linear & 42.2092714 & 0.00066 \\
& Quadratic & 12.776613 & 0.003229 \\
EAS & Cubic & -2.6449157 & 0.238605 \\
SAP & Intercept & 45.6779915 & 0.000448 \\
& Quadratic & 13.1334601 & 0.017138 \\
& Cubic & -6.4551024 & 0.153148 \\
\hline \multirow{2}{*}{ ADM } & Intercept & 41.0370773 & 0.000328 \\
& Linear & 48.9162845 & 0.001238 \\
& Quadratic & 15.3110781 & 0.004849 \\
& Cubic & -6.6908573 & 0.084484 \\
\hline
\end{tabular}

Table 3. $R^{2}$, Adjusted $R^{2}$ and Standard error $S$ of models

\begin{tabular}{ccccc}
\hline \multirow{2}{*}{ Skill } & Model & $R^{2}$ & Adjusted $R^{2}$ & $S$ \\
\hline \multirow{2}{*}{ Testing } & Linear & 0.948 & 0.938 & 10.564 \\
& Quadratic & 0.997 & 0.995 & 2.949 \\
\hline EAS & Linear & 0.954 & 0.945 & 10.647 \\
SAP & Quadratic & 0.989 & 0.983 & 5.958 \\
\hline \multirow{2}{*}{ ADM } & Linear & 0.926 & 0.911 & 12.701 \\
& Quadratic & 0.988 & 0.983 & 5.642 \\
\hline
\end{tabular}

Table 4. F statistics and p values of ANOVA

\begin{tabular}{ccc}
\hline Skill & $\mathrm{F}$ & p value \\
\hline Testing & 614.8110 & 0.0000 \\
EAS SAP & 172.2530 & 0.0001 \\
ADM & 169.7578 & 0.0001
\end{tabular}


Table 5. Model coefficients

\begin{tabular}{cccc}
\hline & Testing & EAS SAP & ADM \\
\hline Intercept & 11.2266 & 7.2686 & 19.10677 \\
linear & 0.3558 & 0.7038 & -2.15963 \\
Square & 0.1011 & 0.0954 & 0.21458 \\
\hline
\end{tabular}

Substituting the values of demand, cost and bench strength from Table 1 and coefficients of recruitment lead time models from Table 5 in (1) to (6), the optimization problem became Minimize $\mathrm{z}=$

$640 x_{1}+800 x_{2}+933 x_{3}+4800 x_{4}+2480 x_{5}$

$$
+30 y_{1}+33 y_{2}+42 y_{3}+25 y_{4}+21 y_{5}
$$

Subject to

$$
\begin{aligned}
& x_{1}+y_{1} \geq 30 \\
& x_{2}+y_{2} \geq 28 \\
& x_{3}+y_{3} \geq 22 \\
& x_{4}+y_{4} \geq 8 \\
& x_{5}+y_{5} \geq 12 \\
& 12 y_{4} \leq 30 \\
& 18 y_{5} \leq 30 \\
& x_{1} \leq 65 \\
& x_{2} \leq 43 \\
& x_{3} \leq 32 \\
& x_{4} \leq 39 \\
& x_{5} \leq 37 \\
& x_{i} \geq 0, i=1,2, \ldots, 5 \\
& y_{i} \geq 0, i=1,2, \ldots, 5 \\
& x_{i}=\text { int eger } \\
& y_{i}=\text { int eger }
\end{aligned}
$$$$
11.2166+0.3558 \times y_{1}+0.1011 \times y_{1}^{2} \leq 30
$$$$
7.2686+0.7038 \times y_{2}+0.0954 \times y_{2}^{2} \leq 30
$$$$
19.1068-2.1596 \times y_{3}+0.2146 \times y_{3}^{2} \leq 30
$$

The available time for recruitment $T_{i}, i=1,2, \ldots, 5$ are taken as less than or equal to 30 days since the demand is for the upcoming month. The aforementioned problem is solved using MS Excel solver. The result obtained is discussed in the next session.

\section{Results}

The current practice followed by many Information Technology companies is to keep a portion of the resources on bench to tackle the issue of non-availability of skilled resources whenever required. In this paper, the author proposes a methodology of fulfilling the monthly resource demands by choosing an optimum mix of bench and new recruitments. The methodology is demonstrated through a case study. The optimum solution for the problem obtained using MS Excel solver is given in Table 6 . The Table 6 shows that the optimum bench strength required is only 61 . The total cost (bench strength cost + recruitment cost) to meet the demand is only USD 42,863, which is much lower than the cost of maintaining the existing bench strength of 216 resources. Moreover the optimum bench strength together with number of resources to be recruited would exactly meet the monthly skill wise demands. Hence it is concluded that the proposed methodology of fulfilling the monthly resource demands by choosing an optimum mix of bench and new recruitments is better than the current practice of meeting the demand by maintaining large bench strength.

Table 6. Optimum solution

\begin{tabular}{ccccc}
\hline \multirow{2}{*}{ Skill } & \multicolumn{3}{c}{ Optimum Solution } & \\
\cline { 2 - 4 } & $\begin{array}{c}\text { Bench } \\
\text { Strength }\end{array}$ & $\begin{array}{c}\text { New } \\
\text { Recruitment }\end{array}$ & Total & \\
\hline Testing & 19 & 11 & 30 & 30 \\
EAS & 16 & 12 & 28 & 28 \\
SAP & & & & \\
ADM & 9 & 13 & 22 & 22 \\
.Net & 6 & 2 & 8 & 8 \\
Java & 11 & 1 & 12 & 12 \\
Total & 61 & 39 & 100 & 139 \\
\hline
\end{tabular}

\section{Conclusions}

The most important risk in IT projects is the nonavailability of resources with the correct skills at the right time. The IT companies mitigate this risk by maintaining a portion of the resources with various skills on bench. This adds cost to the company. In this paper the author suggests a methodology for reducing the bench strength without increasing the risk of not having the required number of skilled resources to meet the demand. The suggested methodology is to obtain an optimum mix of resources from bench and through recruitment. This will drastically reduce the cost of maintaining large bench strength.

The problem is formulated as an integer programming problem. The objective of the problem is to minimize the total cost, which includes the cost of maintaining the bench strength and recruitment cost. The solution also has to meet the constraints on the demand, availability of resources on bench and the recruitment lead time.

The suggested methodology is demonstrated using a case study from a medium scale Indian IT company. The monthly demand of the company for resources with various skill sets namely Testing, EAS SAP, ADM,.Net 
and Java has been formulated as an integer programming problem and solved using MS Excel solver. The optimum solution showed that only 61 resources are required in the bench to meet the demand of 100 resources and the remaining resources can be recruited within the specified time. The company has been maintaining 216 resources on bench, hence a reduction of bench strength by 155 resources. The cost of maintaining 61 resources on bench and recruiting the remaining resources is only USD 42,863 while the cost of maintaining 216 resources on bench is USD 142,347. Hence it is concluded that the proposed methodology is superior to the current practice of maintaining large bench strength of resources.

The problem can be formulated for any number of skill sets to meet the demands for any specified time period (monthly, quarterly, half yearly, etc.). Moreover the problem can be easily solved using widely available MS Excel solver utility, which is part of the Microsoft office.

\section{References}

Al-Yakoob, S. M. and Sherali, H. D. (2007). Mixedinteger programming models for an employee scheduling problem with multiple shifts and work locations. Annals of Operations Research, 155(1), 119-142.

Atamtürk, A. and Savelsbergh, M. W. (2005). Integerprogramming software systems. Annals of Operations Research, 140(1), 67-124.

Boby, J. and Kadadevaramath, R.S. (2013). Optimization of the yield of the code review process. Proceedings of the International Conferences on Quality, Reliability and Operations Research, Mumbai, India, 161-167.

Bouras, A. and Bendak, S. (2014). Managing IT projects in public companies: A case study. Journal of Engineering, Project, and Production Management, 4(2), 74-80.

Brezulianu, A., Fira, M., and Fira, L. (2009). A genetic algorithm approach for a constrained employee scheduling problem as applied to employees at mall type shops. Proceedings of the 2009 International Conference on Hybrid Information Technology, 497501. ACM.

Brown, A. M. (2006). A non-linear regression analysis program for describing electrophysiological data with multiple functions using Microsoft Excel. Computer methods and programs in biomedicine, 82(1), 51-57.

Chua, D.K.H., Kog, Y.C. and Loh, P. K. (1999). Critical success factors for different project objectives. Journal of construction engineering and management, 125 (3), 142-150.

Coelho, L. D. S. (2009). An efficient particle swarm approach for mixed-integer programming in reliability-redundancy optimization applications. Reliability Engineering \& System Safety, 94(4), 830837.

Crawford, S. L. (2006). Correlation and regression. Circulation, 114(19), 2083-2088.

Crawley, M. J. (2012). The R book. John Wiley \& Sons.

Czarnigowska, A. and Sobotka, A. (2014). Estimating construction duration for public roads during the preplanning phase. Journal of Engineering, Project \& Production Management, 4(1), 26-35.
Dasgupta, P. K. (2008). Chromatographic peak resolution using Microsoft Excel Solver: The merit of time shifting input arrays. Journal of Chromatography A, 1213(1), 50-55.

Draper, N. R. and Smith, H. (2003). Applied Regression Analysis. John Wiley \& Sons.

Fang, X., Jiang, S. D., Raut, K., and Qiu, J. W. (2006). Applications of Microsoft Excel Solver function in water resource engineering. Proceedings of the Texas ASCE Spring Conference, Beaumont, Texas, 19-22.

Floudas, C. A. and Lin, X. (2005). Mixed integer linear programming in process scheduling: Modeling, algorithms, and applications. Annals of Operations Research, 139(1), 131-162.

Fylstra, D., Lasdon, L.,Watson, J. and Waren, A. (1999). Design and use of the Microsoft excel solver, Interfaces, 28(5), 29 - 55.

Hillier, F. S. and Lieberman, G. J. (2008). Operations Research - Concepts and Cases. Tata McGraw-Hill Publishing Company Ltd.

Huq, F., Cutright, K., and Martin, C. (2004). Employee scheduling and makespan minimization in a flow shop with multi-processor work stations: a case study. Omega, 32(2), 121-129.

Jalote, P. (2000). CMM in practice: Process for executing software projects at Infosys. Addison-Wesley Longman Inc.

Lennartsson, M. and Björnfot, A. (2012). Production resource management in the industrialised housebuilding supply chain, Journal of Engineering, Project, and Production Management, 2(2), 787-87.

Li, D. and Sun, X. (2006). Nonlinear integer programming. Springer.

Mohan, S. (2008). Scheduling part-time personnel with availability restrictions and preferences to maximize employee satisfaction. Mathematical and Computer Modelling, 48(11), 1806-1813.

Montgomery, D. C. and Runger, G. C. (2007). Applied statistics and probability for engineers. Wiley - India.

Montgomery, D. C., Peck, E. A. and Vining, G. G. (2012). Introduction to linear regression analysis. John Wiley \& Sons.

Morton, D. P. and Popova, E. (2004). A Bayesian stochastic programming approach to an employee scheduling problem. Iie Transactions, 36(2), 155-167.

Narayanan, S. and Swaminathan, J. M. (2007). Information Technology Off shoring to India: Pitfalls, Opportunities and Trends. Retrieved from http:// public.kenan-flagler.unc.edu / Faculty/ swaminaj/ research/ paper/ NS2.pdf.

Nguyen, Q.T. and Chua, D.K.H. (2014). Criticality of schedule constraints - classification and identification for Project Management, Journal of Engineering, Project, and Production Management, 4(1), 17-25.

Porananond, D. and Thawesaengskulthai, N. (2014). Risk management for new product development projects in food industry. Journal of Engineering, Project, and Production Management, 4(2), 99-113.

Souliman, M. I., Mamlouk, M. S., El-Basyouny, M. M., and Zapata, C. E. (2010). Calibration of the AASHTO MEPDG for flexible pavement for arizona conditions. Proceedings of the Transportation Research Board 89th Annual Meeting, Washington DC, No. 10-1817. 
Taha, H. A. (1987). Operations Research - An Introduction. Macmillan Publishing Company.

Zhao, Y. (2012). R and Data Mining: Examples and Case Studies. Academic Press.

Zou, K. H., Tuncali, K., and Silverman, S. G. (2003). Correlation and Simple linear regression 1. Radiology, 227(3), 617-628.

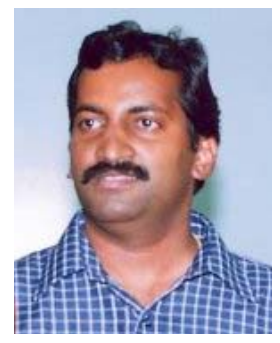

Boby John is currently a Technical Officer at Statistical Quality Control \& Operations Research (SQC \& OR) Unit, Indian Statistical Institute, Bangalore. He received Master of Science degree in Statistics from the Mahatma Gandhi University, Kottyam, India and Master of Technology degree in Quality, Reliability \& Operations Research from Indian Statistical Institute, Kolkata. He is engaged in teaching \& consulting in the fields of Six Sigma, Quality \& Reliability Engineering, Business Analytics, Process Performance Modeling, etc. He has published more than ten papers in international journals and presented many papers in international conferences. 